\title{
Several Schur complement inequalities on block Hadamard product
}

\author{
Mustafa Ozel ${ }^{1}$ and Ayca Ileri ${ }^{2}$ \\ ${ }^{1}$ Dokuz Eylul University, Faculty of Engineering, Department of Geophysics, Buca, Izmir, Turkey \\ ${ }^{2}$ Dokuz Eylul University, Graduate School of Natural and Applied Sciences, Buca, Izmir, Turkey
}

Received: 19 September 2017, Accepted: 7 November 2017

Published online: 25 December 2017.

\begin{abstract}
The Schur complement theory is very important in many areas such as statistics, matrix analysis, numerical analysis, and control theory. It is a powerful tool to discuss many significant results. This paper deals with the inequalities involving block Hadamard product of positive definite matrices. By using the definition and the properties of block Hadamard product, we obtain useful inequalities on the Schur complement of the block Hadamard product of two positive definite matrices and their inverses. Finally, we give some numerical examples which confirm our theoritical analysis.
\end{abstract}

Keywords: Block matrices, block Hadamard product, Schur complement, positive definite matrices.

\section{Introduction}

Let $M_{m, n}$ be the space of $m \times n$ matrices with complex entries and $\boldsymbol{M}_{p, q}\left(M_{m, n}\right)$ be the space of $p \times q$ block matrices $\boldsymbol{A}=\left(A_{\alpha, \beta}\right)_{\alpha=1, \ldots, p}^{\beta=1, \ldots, q}$ whose $\alpha, \beta$ entry belongs to $M_{m, n}$, for $m, n, p, q \in \mathbb{Z}^{+}$. If $m=n$, then we denote $M_{n, n}$ by $M_{n}$ and $p=q$ we denote $\boldsymbol{M}_{p, q}$ by $\boldsymbol{M}_{p}$. Also we denote the $p n \times p n$ identity matrix as $\boldsymbol{I}_{p}$. If $\boldsymbol{A}>0$, we say that $\boldsymbol{A}$ is positive definite, and if $\boldsymbol{A}>\boldsymbol{B}$ or $\boldsymbol{B}<\boldsymbol{A}$, we say that $\boldsymbol{A}-\boldsymbol{B}>0$ for positive definite matrices $\boldsymbol{A}$ and $\boldsymbol{B}$.

Let $\boldsymbol{\alpha} \subset\{1,2, \ldots, p\}, \boldsymbol{\beta} \subset\{1,2, \ldots, q\}$ be the index sets and $\boldsymbol{\alpha}^{c}=\{1,2, \ldots, p\} \backslash \boldsymbol{\alpha}, \boldsymbol{\beta}^{c}=\{1,2, \ldots, q\} \backslash \boldsymbol{\beta}$ be the complements of $\boldsymbol{\alpha}$ and $\boldsymbol{\beta}$, and their cardinalities are $|\boldsymbol{\alpha}|$ and $|\boldsymbol{\beta}|$.

Let $\boldsymbol{A}(\boldsymbol{\alpha}, \boldsymbol{\beta})$ denote the block submatrix of $\boldsymbol{A}$ with the block rows indexed by $\boldsymbol{\alpha}$ and the block columns indexed by $\boldsymbol{\beta}$. As usual we write $\boldsymbol{A}(\boldsymbol{\alpha})$ for $\boldsymbol{A}(\boldsymbol{\alpha}, \boldsymbol{\alpha})$.

By using the definition of Schur complement introduced in [4], we will give the block form of the Schur complement as follows:

If $|\boldsymbol{\alpha}|=|\boldsymbol{\beta}|$ and $\boldsymbol{A}(\boldsymbol{\alpha}, \boldsymbol{\beta})$ is nonsingular then the block of the Schur complement of $\boldsymbol{A}(\boldsymbol{\alpha}, \boldsymbol{\beta})$ in $\boldsymbol{A}$ is

$$
A / A(\boldsymbol{\alpha}, \boldsymbol{\beta})=A\left(\boldsymbol{\alpha}^{c}, \boldsymbol{\beta}^{c}\right)-A\left(\boldsymbol{\alpha}^{c}, \boldsymbol{\beta}\right)(A(\boldsymbol{\alpha}, \boldsymbol{\beta}))^{-1} A\left(\boldsymbol{\alpha}, \boldsymbol{\beta}^{c}\right)
$$

It is often convenient to write $A / \alpha$ for $A / A(\alpha)$. 
Definition 1. Let $\boldsymbol{A}=\left(A_{\alpha \beta}\right), \boldsymbol{B}=\left(B_{\alpha \beta}\right) \in \boldsymbol{M}_{p, q}$ where each block is an $n \times n$ matrix with complex entries. The block Hadamard product of $\boldsymbol{A}$ and $\boldsymbol{B}$ is

$$
\boldsymbol{A} \square \boldsymbol{B}=\left(A_{\alpha \beta} B_{\alpha \beta}\right)_{\alpha=1, \ldots, p}^{\beta=1, \ldots, q}
$$

where $A_{\alpha \beta} B_{\alpha \beta}$ denotes the usual matrix product [2,3].

If every $n \times n$ block of $\boldsymbol{A}$ commutes with every $n \times n$ block of $\boldsymbol{B}$ then we call these matrices block commuting and we denote this by $\boldsymbol{A}_{b c} \boldsymbol{B}$.

Gunter and Klotz [1] in Proposition 3.4 proved the inequality $(\boldsymbol{A} \square \boldsymbol{B})^{-1} \leq \boldsymbol{A}^{-1} \square \boldsymbol{B}^{-1}$. Through the following lemma, we will prove this inequality in a different way.

\section{Theory and main results}

Lemma 1. Let $\boldsymbol{A}, \boldsymbol{B} \in \boldsymbol{M}_{q}\left(M_{n}\right)$ and $\boldsymbol{C}, \boldsymbol{D} \in \boldsymbol{M}_{p, q}\left(M_{n}\right)$ be positive definite matrices such that $\boldsymbol{A}_{b c} \boldsymbol{B}$ and $\boldsymbol{C}_{b c} \boldsymbol{D}$. Then

$$
(\boldsymbol{C} \square \boldsymbol{D})(\boldsymbol{A} \square \boldsymbol{B})^{-1}(\boldsymbol{C} \square \boldsymbol{D})^{*} \leq\left(\boldsymbol{C A}^{-1} \boldsymbol{C}^{*}\right) \square\left(\boldsymbol{D} \boldsymbol{B}^{-1} \boldsymbol{D}^{*}\right) .
$$

In particular

$$
(\boldsymbol{A} \square \boldsymbol{B})^{-1} \leq \boldsymbol{A}^{-1} \square \boldsymbol{B}^{-1}
$$

and

$$
(\boldsymbol{C} \square \boldsymbol{D})(\boldsymbol{C} \square \boldsymbol{D})^{*} \leq\left(\boldsymbol{C} \boldsymbol{C}^{*}\right) \square\left(\boldsymbol{D} \boldsymbol{D}^{*}\right)
$$

Proof. Let

$$
\hat{\boldsymbol{A}}=\left(\begin{array}{cc}
\boldsymbol{A} & \boldsymbol{C}^{*} \\
\boldsymbol{C} & \boldsymbol{C A}^{-1} \boldsymbol{C}^{*}
\end{array}\right), \quad \hat{\boldsymbol{B}}=\left(\begin{array}{cc}
\boldsymbol{B} & \boldsymbol{D}^{*} \\
\boldsymbol{D} & \boldsymbol{D} \boldsymbol{B}^{-1} \boldsymbol{D}^{*}
\end{array}\right)
$$

Then $\hat{\boldsymbol{A}}$ and $\hat{\boldsymbol{B}}$ are positive semidefinite and

$$
\hat{\boldsymbol{A}} \square \hat{\boldsymbol{B}}=\left(\begin{array}{cc}
\boldsymbol{A} \square \boldsymbol{B} & \boldsymbol{C}^{*} \square \boldsymbol{D}^{*} \\
\boldsymbol{C} \square \boldsymbol{D} & \left(\boldsymbol{C A}^{-1} \boldsymbol{C}^{*}\right) \square\left(\boldsymbol{D B}^{-1} \boldsymbol{D}^{*}\right)
\end{array}\right) \geq 0
$$

Taking the Schur complement of $(1,1)$ block in $\hat{\boldsymbol{A}} \square \hat{\boldsymbol{B}}$

$$
\left(\boldsymbol{C A}^{-1} \boldsymbol{C}^{*}\right) \square\left(\boldsymbol{D} \boldsymbol{B}^{-1} \boldsymbol{D}^{*}\right)-(\boldsymbol{C} \square \boldsymbol{D})(\boldsymbol{A} \square \boldsymbol{B})^{-1}\left(\boldsymbol{C}^{*} \square \boldsymbol{D}^{*}\right) \geq 0
$$

and

$$
(\boldsymbol{C} \square \boldsymbol{D})(\boldsymbol{A} \square \boldsymbol{B})^{-1}\left(\boldsymbol{C}^{*} \square \boldsymbol{D}^{*}\right) \leq\left(\boldsymbol{C} \boldsymbol{A}^{-1} \boldsymbol{C}^{*}\right) \square\left(\boldsymbol{D} \boldsymbol{B}^{-1} \boldsymbol{D}^{*}\right)
$$

are obtained. By using the block form in [3] and choosing the matrices $\boldsymbol{C}$ and $\boldsymbol{D}$ as the identity matrix $\boldsymbol{I}_{\boldsymbol{q}}$ in the inequality (1), we have

$$
(\boldsymbol{A} \square \boldsymbol{B})^{-1} \leq \boldsymbol{A}^{-1} \square \boldsymbol{B}^{-1}
$$

Similarly, when we write $\boldsymbol{I}_{\boldsymbol{q}}$ instead of $\boldsymbol{A}$ and $\boldsymbol{B}$ in (1),

$$
(\boldsymbol{C} \square \boldsymbol{D})(\boldsymbol{C} \square \boldsymbol{D})^{*} \leq\left(\boldsymbol{C} \boldsymbol{C}^{*}\right) \square\left(\boldsymbol{D} \boldsymbol{D}^{*}\right)
$$


is obtained.

Theorem 1. Let $\boldsymbol{A}, \boldsymbol{B} \in \boldsymbol{M}_{p}\left(M_{n}\right)$ be positive definite matrices such that $\boldsymbol{A}_{b c} \boldsymbol{B}$. Then

$$
(\boldsymbol{A} \square \boldsymbol{B}) / \boldsymbol{\alpha} \geq \boldsymbol{A} / \boldsymbol{\alpha} \square \boldsymbol{B} / \boldsymbol{\alpha} .
$$

Proof. Consider the partition

$$
A=\left(\begin{array}{ll}
A_{1} & A_{2} \\
A_{3} & A_{4}
\end{array}\right) \quad B=\left(\begin{array}{ll}
B_{1} & B_{2} \\
B_{3} & B_{4}
\end{array}\right)
$$

and

$$
A \square B=\left(\begin{array}{c}
A_{1} \square B_{1} A_{2} \square B_{2} \\
A_{3} \square B_{3} A_{4} \square B_{4}
\end{array}\right) .
$$

Since there exists a permutation matrix $U$ such that $U^{T} A U / A(\alpha)=A / \alpha$, we use $A / \alpha=A / A_{1}$ and $B / \alpha=B / B_{1}$ and $(\boldsymbol{A} \square \boldsymbol{B}) / \boldsymbol{\alpha}=(\boldsymbol{A} \square \boldsymbol{B}) / \boldsymbol{A}_{1} \square \boldsymbol{B}_{1}$. Then

$$
(A \square B) / A_{1} \square B_{1}=A_{4} \square B_{4}-\left(A_{3} \square B_{3}\right)\left(A_{1} \square B_{1}\right)^{-1}\left(A_{2} \square B_{2}\right)
$$

by the Lemma 1 we get

$$
(A \square B) / A_{1} \square B_{1} \geq A_{4} \square B_{4}-\left(A_{3} A_{1}^{-1} A_{2}\right) \square\left(B_{3} B_{1}^{-1} B_{2}\right)
$$

and since

$$
\begin{aligned}
A_{3} A_{1}^{-1} A_{2}=A_{4}- & A / A_{1} \geq 0 \quad B_{3} B_{1}^{-1} B_{2}=B_{4}-B / B_{1} \geq 0 \\
(A \square B) / A_{1} \square B_{1} & \geq A_{4} \square B_{4}-\left(A_{3} A_{1}^{-1} A_{2}\right) \square\left(B_{3} B_{1}^{-1} B_{2}\right) \\
& =A_{4} \square B_{4}-\left(A_{4}-A / A_{1}\right) \square\left(B_{4}-B / B_{1}\right) \\
& =\left(A / A_{1}\right) \square B_{4}+\left(A_{4}-A / A_{1}\right) \square\left(B / B_{1}\right) \\
& \geq\left(A / A_{1} \square B_{4}\right) \\
& \geq\left(A / A_{1}\right) \square\left(B / B_{1}\right)
\end{aligned}
$$

and therefore, (4) follows.

Corollary 1. Let $\boldsymbol{A}, \boldsymbol{B} \in \boldsymbol{M}_{p}\left(M_{n}\right)$ be positive definite matrices such that $\boldsymbol{A}_{b c} \boldsymbol{B}$. Then

(i) $(\boldsymbol{A} \square \boldsymbol{B})^{-1} / \boldsymbol{\alpha} \leq[(\boldsymbol{A} \square \boldsymbol{B}) / \boldsymbol{\alpha}]^{-1} \leq(\boldsymbol{A} / \boldsymbol{\alpha} \square \boldsymbol{B} / \boldsymbol{\alpha})^{-1} \leq(\boldsymbol{A} / \boldsymbol{\alpha})^{-1} \square(\boldsymbol{B} / \boldsymbol{\alpha})^{-1}$

(ii) $(\boldsymbol{A} \square \boldsymbol{B})^{-1} / \boldsymbol{\alpha} \leq\left(\boldsymbol{A}^{-1} / \boldsymbol{\alpha}\right) \square\left(\boldsymbol{B}^{-1} / \boldsymbol{\alpha}\right) \leq(\boldsymbol{A} / \boldsymbol{\alpha})^{-1} \square(\boldsymbol{B} / \boldsymbol{\alpha})^{-1}$

Proof. (i) Since $\boldsymbol{A}^{-1} / \boldsymbol{\alpha} \leq(\boldsymbol{A} / \boldsymbol{\alpha})^{-1}$, then

$$
(\boldsymbol{A} \square \boldsymbol{B})^{-1} / \boldsymbol{\alpha} \leq[(\boldsymbol{A} \square \boldsymbol{B}) / \boldsymbol{\alpha}]^{-1} .
$$

The second inequality

$$
[(\boldsymbol{A} \square \boldsymbol{B}) / \boldsymbol{\alpha}]^{-1} \leq(\boldsymbol{A} / \boldsymbol{\alpha} \square \boldsymbol{B} / \boldsymbol{\alpha})^{-1}
$$

follows from Theorem 1 . The last inequality is a consequence of Lemma 1 .

(ii) Let us partition $\boldsymbol{A}$ and $\boldsymbol{B}$ as in Lemma 1 . To avoid the confusion, we shall use the notation $\widetilde{\boldsymbol{A}_{\mathbf{1}} \square \boldsymbol{B}_{1}}$, that is used in [3], in place of $(\boldsymbol{A} \square \boldsymbol{B}) / \boldsymbol{\alpha}$, which is the Schur complement of $(1,1)$ block of $\boldsymbol{A} \square \boldsymbol{B}$ and $\widetilde{\boldsymbol{A}_{1}}, \widetilde{\boldsymbol{B}_{1}}$ are the Schur complements of 
the $(1,1)$ blocks of $\boldsymbol{A}, \boldsymbol{B}$. Similarly, $\widetilde{\boldsymbol{A}_{\mathbf{4}}}, \widetilde{\boldsymbol{B}_{\mathbf{4}}}$ and $\widetilde{\boldsymbol{A}_{\mathbf{4}} \square \boldsymbol{B}_{\mathbf{4}}}$ mean that the Schur complement of $(2,2)$ block of $\boldsymbol{A}, \boldsymbol{B}$ and $\boldsymbol{A} \square \boldsymbol{B}$, respectively. Hence

$$
\boldsymbol{A}^{-1}=\left(\begin{array}{cc}
{\widetilde{\boldsymbol{A}_{4}}}^{-1} & * \\
* & {\widetilde{\boldsymbol{A}_{1}}}^{-1}
\end{array}\right), \quad \boldsymbol{B}^{-1}=\left(\begin{array}{cc}
{\widetilde{\boldsymbol{B}_{4}}}^{-1} & * \\
* & {\widetilde{\boldsymbol{B}_{1}}}^{-1}
\end{array}\right)
$$

and

$$
(\boldsymbol{A} \square \boldsymbol{B})^{-1}=\left(\begin{array}{cc}
\left(\widehat{\boldsymbol{A}_{\mathbf{4}} \square \boldsymbol{B}_{\mathbf{4}}}\right)^{-1} & * \\
* & \left(\widehat{\boldsymbol{A}_{\mathbf{1}} \square \boldsymbol{B}_{1}}\right)^{-1}
\end{array}\right)
$$

where * denotes entries irrelevant to our discussion, that is

$$
\left.\left(\widetilde{\boldsymbol{A}_{\mathbf{4}} \square \boldsymbol{B}_{\mathbf{4}}}\right)^{-1}=(\boldsymbol{A} \square \boldsymbol{B})_{11}^{-1}, \quad \widetilde{\widetilde{\boldsymbol{A}_{\mathbf{4}} \square \boldsymbol{B}_{\mathbf{4}}}}\right)^{-1}=\left(\widetilde{\boldsymbol{A} \square \boldsymbol{B})_{11}^{-1}}\right.
$$

or

$$
\begin{aligned}
& {\widetilde{\left(\boldsymbol{A}_{\mathbf{4}} \square \boldsymbol{B}_{4}\right.}}^{-1}=\boldsymbol{A}_{4} \square \boldsymbol{B}_{4} . \\
& \left.\widetilde{\left(\boldsymbol{A}_{\mathbf{4}} \square \boldsymbol{B}_{4}\right.}\right)^{-1}=\left(\boldsymbol{A}_{\mathbf{4}} \square \boldsymbol{B}_{\mathbf{4}}\right)^{-1} \leq \boldsymbol{A}_{\mathbf{4}}^{-1} \square \boldsymbol{B}_{\mathbf{4}}^{-1} \\
& =\widetilde{{\widetilde{A_{4}}}^{-1}} \square \widetilde{{\widetilde{B_{4}}}^{-1}} \\
& =\left(\widetilde{\boldsymbol{A}^{-1}}\right)_{1} \square\left(\widetilde{\boldsymbol{B}^{-1}}\right)_{1} \\
& =\left(\boldsymbol{A}^{-1} / \boldsymbol{\alpha}\right) \square\left(\boldsymbol{B}^{-1} / \boldsymbol{\alpha}\right) \text {. }
\end{aligned}
$$

By using Theoerem 1,

$$
\left(\boldsymbol{A}^{-1} / \boldsymbol{\alpha}\right) \square\left(\boldsymbol{B}^{-1} / \boldsymbol{\alpha}\right) \leq\left(\boldsymbol{A}^{-1} \square \boldsymbol{B}^{-1}\right) / \boldsymbol{\alpha}
$$

is written. For the last inequality, we partition $\boldsymbol{A}^{-1} \square \boldsymbol{B}^{-1}$ as follows

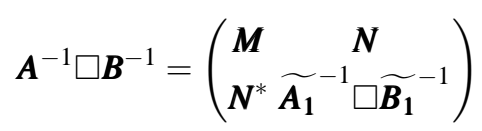

$\boldsymbol{M}$ and $\boldsymbol{N}$ any matrices. Then

$$
\begin{aligned}
\left(\boldsymbol{A}^{-1} \square \boldsymbol{B}^{-1}\right) / \boldsymbol{\alpha} & =\left(\widetilde{\boldsymbol{A}^{-1} \square \boldsymbol{B}^{-1}}\right)_{1} \\
& ={\widetilde{\boldsymbol{A}_{1}}}^{-1} \square \widetilde{\boldsymbol{B}_{1}}-\boldsymbol{N}^{*} \boldsymbol{M}^{-1} \boldsymbol{N} \\
& \leq{\widetilde{\boldsymbol{A}_{1}}}^{-1} \square \widetilde{\boldsymbol{B}_{1}} \\
& =(\boldsymbol{A} / \boldsymbol{\alpha})^{-1} \square(\boldsymbol{B} / \boldsymbol{\alpha})^{-1}
\end{aligned}
$$

Corollary 2. Let $\boldsymbol{A}>0, \boldsymbol{B}>0 \in \boldsymbol{M}_{p}\left(M_{n}\right)$ and $\boldsymbol{A}_{b c} \boldsymbol{B}$. Then

$$
[(\boldsymbol{A} \square \boldsymbol{B}) / \boldsymbol{\alpha}]^{-1} \leq\left(\boldsymbol{A}^{-1} \square \boldsymbol{B}^{-1}\right) / \boldsymbol{\alpha}
$$

Proof. According to Theorem 1,

$$
\left[(\boldsymbol{A} \square \boldsymbol{B}) / \boldsymbol{A}_{1} \square \boldsymbol{B}_{1}\right] \geq\left(\boldsymbol{A} / \boldsymbol{A}_{1}\right) \square \boldsymbol{B}_{4}, t
$$


then

$$
\begin{aligned}
{\left[(\boldsymbol{A} \square \boldsymbol{B}) / \boldsymbol{A}_{1} \square \boldsymbol{B}_{1}\right]^{-1} } & \leq\left[\left(\boldsymbol{A} / \boldsymbol{A}_{1}\right) \square \boldsymbol{B}_{4}\right]^{-1} \\
& =\left[\left(\boldsymbol{A} / \boldsymbol{A}_{1}\right) \square\left(\boldsymbol{B}^{-1} / \boldsymbol{B}_{1}\right)^{-1}\right]^{-1} \\
& \leq\left(\boldsymbol{A} / \boldsymbol{A}_{1}\right)^{-1} \square\left(\boldsymbol{B}^{-1} / \boldsymbol{B}_{1}\right) \\
& =\boldsymbol{A}_{4} \square\left(\boldsymbol{B}^{-1} / \boldsymbol{B}_{1}\right) \\
& \leq \boldsymbol{A}_{4} \square\left(\boldsymbol{B}^{-1} / \boldsymbol{B}_{1}\right)+\boldsymbol{A}_{4} \square\left[\left(\boldsymbol{B} / \boldsymbol{B}_{1}\right)^{-1}-\boldsymbol{B}^{-1} / \boldsymbol{B}_{1}\right] .
\end{aligned}
$$

Since $\boldsymbol{A}_{b c} \boldsymbol{B}$, the right hand side of the last inequality equals

$$
\left(\boldsymbol{B}^{-1} / \boldsymbol{B}_{1}\right) \square \boldsymbol{A}_{4}+\left[\left(\boldsymbol{B} / \boldsymbol{B}_{1}\right)^{-1}-\boldsymbol{B}^{-1} / \boldsymbol{B}_{1}\right] \square \boldsymbol{A}_{4} .
$$

Then

$$
\left[(\boldsymbol{A} \square \boldsymbol{B}) / \boldsymbol{A}_{1} \square \boldsymbol{B}_{1}\right]^{-1} \leq\left(\boldsymbol{B}^{-1} / \boldsymbol{B}_{1}\right) \square \boldsymbol{A}_{4}+\left[\left(\boldsymbol{B} / \boldsymbol{B}_{1}\right)^{-1}-\boldsymbol{B}^{-1} / \boldsymbol{B}_{1}\right] \square \boldsymbol{A}_{4} \leq\left(\boldsymbol{A}^{-1} \square \boldsymbol{B}^{-1}\right) / \boldsymbol{A}_{1} \square \boldsymbol{B}_{1}
$$

This completes the proof.

Example 1. Suppose that $\boldsymbol{A}, \boldsymbol{B} \in \boldsymbol{M}_{2}\left(M_{2}\right)$ be positive definite matrices such that

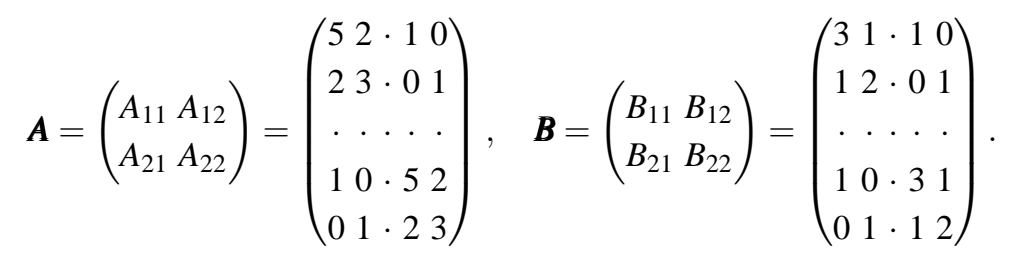

For the Theorem 1, let

$$
\boldsymbol{\alpha}=\{1\}, \quad \boldsymbol{\beta}=\{1\}, \quad \boldsymbol{\alpha}^{c}=\{2\}, \quad \boldsymbol{\beta}^{c}=\{2\} .
$$

By using this partition, we find that

$$
(\boldsymbol{A} \square \boldsymbol{B}) / \boldsymbol{\alpha}-\boldsymbol{A} / \boldsymbol{\alpha} \square \boldsymbol{B} / \boldsymbol{\alpha}=\left(\begin{array}{c}
107 / 5524 / 55 \\
24 / 5583 / 55
\end{array}\right)
$$

is a hermitian matrix with eigenvalues $\lambda_{1}=1.2394, \lambda_{2}=2.2151$ and the proof is completed.

Example 2. Let us apply the Corollary 1 and 2 using the matrices in the Example ??. By computations we find that

$$
\begin{array}{cc}
{[(\boldsymbol{A} \square \boldsymbol{B}) / \boldsymbol{\alpha}]^{-1}=\left(\begin{array}{cc}
47 / 279 & -56 / 279 \\
-56 / 279 & 103 / 279
\end{array}\right),} & (\boldsymbol{A} \square \boldsymbol{B})^{-1} / \boldsymbol{\alpha}=\left(\begin{array}{c}
8 / 55-9 / 55 \\
-9 / 5517 / 55
\end{array}\right) \\
(\boldsymbol{A} / \boldsymbol{\alpha})^{-1} \square(\boldsymbol{B} / \boldsymbol{\alpha})^{-1}=\left(\begin{array}{cc}
17 / 44 & -6 / 11 \\
-6 / 11 & 41 / 44
\end{array}\right), & (\boldsymbol{A} / \boldsymbol{\alpha} \square \boldsymbol{B} / \boldsymbol{\alpha})^{-1}=\left(\begin{array}{c}
17 / 44-6 / 11 \\
-6 / 1141 / 44
\end{array}\right) \\
\left(\boldsymbol{A}^{-1} / \boldsymbol{\alpha}\right) \square\left(\boldsymbol{B}^{-1} / \boldsymbol{\alpha}\right)=\left(\begin{array}{cc}
8 / 55 & -9 / 55 \\
-9 / 55 & 17 / 55
\end{array}\right), & \left(\boldsymbol{A}^{-1} \square \boldsymbol{B}^{-1}\right) / \boldsymbol{\alpha}=\left(\begin{array}{c}
18 / 55-9 / 20 \\
-9 / 20171 / 220
\end{array}\right)
\end{array}
$$




$$
(\boldsymbol{A} \square \boldsymbol{B})^{-1}=\left(\begin{array}{cccc}
47 / 279 & -56 / 279 & -16 / 279 & 25 / 279 \\
-56 / 279 & 103 / 279 & 25 / 279 & -41 / 279 \\
-16 / 279 & 25 / 279 & 47 / 279 & -56 / 279 \\
25 / 279 & -41 / 279 & -56 / 279 & 103 / 279
\end{array}\right) .
$$

Since the matrix

$$
[(\boldsymbol{A} \square \boldsymbol{B}) / \boldsymbol{\alpha}]^{-1}-(\boldsymbol{A} \square \boldsymbol{B})^{-1} / \boldsymbol{\alpha}=\left(\begin{array}{cc}
0.023004 & -0.037081 \\
-0.037081 & 0.060085
\end{array}\right)
$$

is a hermitian matrix with eigenvalues $\lambda_{1}=0.000087$ and $\lambda_{2}=0.083002$, the first inequality holds $(i)$. Similarly, the second and third inequalities are verified. The accuracy of the inequalities in (ii) can be easily seen from the above calculations.

Furthermore, we obtain that

$$
\left(\boldsymbol{A}^{-1} \square \boldsymbol{B}^{-1}\right) / \boldsymbol{\alpha}-[(\boldsymbol{A} \square \boldsymbol{B}) / \boldsymbol{\alpha}]^{-1}=\left(\begin{array}{cc}
0.159 & -0.249 \\
-0.249 & 0.408
\end{array}\right)>0
$$

is a Hermitian matrix with eigenvalues $\lambda_{1}=0.0051 \lambda_{2}=0.5619$, this result gives the inequality in the Corollary 2 .

In this paper, we investigate the relation between the Schur complement theory and the block Hadamard product to produce various kinds of matrix inequalities. We obtain useful inequalities on the Schur complement of the block Hadamard product of two matrices and their inverses. Future research will focus on improving the inequalities of the eigenvalues and estimation for the lower bound of the minimum eigenvalue of the block Hadamard product of two matrices.

\section{Competing interests}

The authors declare that they have no competing interests.

\section{Authors' contributions}

All authors have contributed to all parts of the article. All authors read and approved the final manuscript.

\section{References}

[1] M. Günther, L. Klotz, Schur's theorem for a block Hadamard product, Linear Algebra and its Applications, 437(2012) 948-956.

[2] R. A. Horn, R. Mathias, and Y. Nakamura, Inequalilities for Unitarily Invariant Norms and Bilinear Matrix Products, Linear and Multilinear Algebra, 30(1991), 303-314.

[3] B. Wang, F. Zhang, Trace and eigenvalue inequaities for ordinary and Hadamard products of positive semidefinite Hermitian matrices, SIAM J. Matrix Anal. Appl., 16(1995) 1173-1183.

[4] F. Zhang, Matrix Theory: Basic results and techniques, Springer, 2011. 